\title{
DIVERSAS PERSPECTIVAS EN RELACIÓN CON ALGUNOS ASPECTOS CONCEPTUALES Y PRÁCTICOS DE LA INCLUSIÓN EDUCATIVA
}

\section{Various Perspectives in Relation to Some Conceptual and Practical Aspects of Educational Inclusion}

\author{
Rafael Félix Bell Rodríguez, Dr. C. \\ Instituto Superior Tecnológico de Formación \\ Profesional Administrativa y Comercial \\ Guayaquil, Ecuador \\ https://orcid.org/0000-0002-0255-642X \\ rafael.bell@formacion.edu.ec
} Palabras claves: Inclusión Educativa, Necesidades Educativas Especiales, Investigación
Inclusiva.

Keywords: Educational Inclusion, Special Educational Needs, Inclusive Research.
Recibido: 23 de mayo de 2018

Aceptado: 15 de junio de 2018

\section{RESUMEN}

En este artículo se abordan algunos aspectos conceptuales y prácticos de la inclusión educativa desde diversas perspectivas con el ánimo de contribuir al diálogo en favor del análisis y de la identificación de los fundamentos y estrategias que permitan propiciar el necesario avance hacia el logro de los objetivos de este complejo proceso. A partir de la revisión de variadas fuentes bibliográficas, entre las que se incluyen algunas publicadas en ruso e inglés respectivamente, se esbozan diferentes aproximaciones e implicaciones de importantes conceptos en este ámbito, subrayando los aspectos positivos de la inclusión para todos los escolares y los riesgos a los que este proceso se enfrenta cuando se acomete su realización sin las condiciones requeridas para su éxito y no se cuenta con el compromiso y la correspondiente preparación de los docentes y de toda la comunidad educativa para su desarrollo. De igual manera se describen distintas aristas en relación con la comprensión de las necesidades educativas especiales, la inclusión educativa y la investigación inclusiva, que reflejan algunos de los principales contenidos del debate conceptual y práctico que tiene lugar en varias de las múltiples problemáticas de la educación inclusiva.

\begin{abstract}
This article addresses some conceptual and practical aspects of educational inclusion from different perspectives with the aim of contributing to the dialogue in favor of analysis and the identification of the foundations and strategies that will facilitate the necessary progress towards the achievement of the objectives of this complex process. From the analysis of various bibliographical sources, including some published in Russian and English respectively, different approaches and implications of important concepts in this field are outlined, highlighting the positive aspects of inclusion for all schoolchildren and the risks to what this process faces when its realization is undertaken without the conditions required for its success and there is no commitment and the corresponding preparation of the teachers and of the entire educational community for its development. In the same way, different aspects are described in relation to the understanding of special educational needs, educational inclusion and inclusive research, which reflect some of the main contents of the conceptual and practical debate that takes place in several of the multiple problems of education inclusive.
\end{abstract}




\section{INTRODUCCIÓN}

Para el análisis de los principios y tendencias de mayor importancia y actualidad en el ámbito pedagógico y educativo a nivel internacional resulta de mucho valor la posibilidad de acceder a diversas fuentes, incluidas aquellas que alcanzan un notable reconocimiento en otras latitudes y a las que, comúnmente, las barreras lingüísticas obstaculizan su acceso en el marco, por ejemplo, de los países de habla hispana. Ese es el caso de la inclusión educativa, tal vez la apuesta de mayor trascendencia para la transformación de los sistemas educativos a escala mundial, en torno a la cual resulta indispensable seguir promoviendo el diálogo y la reflexión de investigadores, docentes, especialistas, personas con discapacidad y de toda la sociedad, generando espacios para el acercamiento a puntos de vista que, como los que se publican en otras lenguas por prestigiosas revistas científicas y libros de destacados autores de otras latitudes, no son muy difundidos fuera de las fronteras de sus respectivos países.

Sin embargo, a pesar de las diferencias determinadas por el efecto de los factores contextuales, no es difícil identificar algunas similitudes teóricas y prácticas con las posturas de los investigadores internacionales, que revelan la naturaleza compleja del objeto de estudio y confirman la necesidad y posibilidades del diálogo científico para propiciar el mejor aprovechamiento del aporte de todos en la superación de los desafíos que la inclusión educativa presupone. En línea con lo señalado el objetivo del presente artículo es exponer algunos aspectos conceptuales y prácticos de la inclusión educativa desde diversas perspectivas como una contribución al diálogo en favor del análisis y de la identificación de fundamentos y estrategias para el necesario avance hacia el cumplimiento de los objetivos de este permanente proceso.

\section{DESARROLLO}

\section{Inclusión educativa, fundamentos, repercusiones y tendencias}

La inclusión educativa de los estudiantes con discapacidad ocupa un reconocido lugar en la concepción y aplicación de las políticas educativas a escala internacional en la actualidad, contando para ello con sólidos argumentos filosóficos, legales, sociales, psicológicos, pedagógicos y, sobre todo, humanos. Desde el punto de vista filosófico se sustenta en el reconocimiento del valor de toda vida humana, de su singularidad como expresión de la diversidad de la que forma parte y del fomento de los valores que son inherentes a la verdadera esencia de la condición humana.

Su fundamentación legal se basa en la consagración y en el ejercicio del derecho de todos los seres humanos a una educación de calidad, cimentada en el respeto y en la igualdad de oportunidades. La perspectiva psicológica se afianza en el papel de la cultura, de la comunicación, de la actividad conjunta, de la mediación y de la interacción con sus familiares y compañeros como fuente para el desarrollo psíquico del niño, en tanto su sustentación pedagógica parte de la proclamación de la inclusión educativa como un principio que ha de regir la concepción y la práctica de la actividad educativa dirigida al logro de la formación integral de la personalidad de todos los estudiantes. Los argumentos mencionados permiten revelar la naturaleza profundamente humana de la inclusión que, entre otros aspectos, se evidencia en sus repercusiones en el estudiantado, en concreto, en los estudiantes que presentan alguna discapacidad y en aquellos en los esa condición no está presente.

En ese sentido, Nazarova, Bogdanova, Prijodko y Yakovleva (2018) destacan, entre los principales aspectos positivos de la inclusión educativa en relación con los estudiantes con discapacidad, los siguientes:

- Generación de contextos y condiciones para la socialización.

- Creación de condiciones para dar respuesta a las necesidades de comunicación.

- Desarrollo de cualidades de la personalidad de los estudiantes, relacionadas con la formación de la autoestima y de la confianza en sí mismo.

- Ampliación de las posibilidades para el enriquecimiento de la experiencia social y comunicativa.

- Incremento de su participación en contextos desde los cuales es posible asumir una posición activa y trascendente para el ejercicio de sus derechos.

Sin embargo, es preciso subrayar que la inclusión educativa es un proceso que implica a todos los estudiantes, y en aquellos que no presentan condición de discapacidad alguna, también se pueden apreciar sus positivas repercusiones, que Nazarova et al. (2018) resume de la siguiente manera:

- Estimulación de la educación del humanismo, de la aceptación de las diferencias, de la solidaridad y de la tolerancia.

- Sensibilización en relación con la necesidad de eliminación de las barreras que en la vida cotidiana obstaculizan la inclusión y valoración del esfuerzo que realizan las personas con discapacidad para su superación. 
- Aprovechamiento de las posibilidades que la inclusión genera para el desarrollo de la capacidad para la interacción con personas con discapacidad, para el aprendizaje de nuevas vías de comunicación e interacción con las personas y con el entorno social en general.

Evidentemente estos no son los únicos aspectos positivos de la inclusión, que por su naturaleza y alcance impacta toda la vida de las instituciones escolares, encerrando grandes potencialidades que, de ser convenientemente aprovechadas, pueden convertirse en un importante catalizador de las transformaciones requeridas para el logro de una educación de calidad para todos. Por ello, como precisan Webster \& Blatchford (2015) la inclusión educativa alcanza cada vez un mayor reconocimiento en diferentes países motivado por razones asociadas a la equidad y a la posibilidad que brinda para el acceso de todos a una educación de la mayor calidad posible con la participación de docentes altamente preparados y el apoyo de los estudiantes. Justamente en esa última precisión consiste una de las tendencias de este proceso a nivel internacional, en el que, junto con ella, también se manifiestan, de acuerdo con Sigal (2016) las siguientes:

- Fortalecimiento de la cooperación internacional y la promoción de iniciativas a distintos niveles a favor del logro de los objetivos de la inclusión.

- Valoración de la importancia del trabajo interdisciplinario y el establecimiento de un enfoque positivo y dinámico en el diagnóstico como una importante premisa para garantizar la mejor respuesta a las necesidades y potencialidades de todos los educandos.

- Aplicación de programas y otras estrategias dirigidas a garantizar la preparación del profesorado y de toda la comunidad educativa para la atención a la diversidad del estudiantado.

- Crecimiento del nivel de conciencia en relación con la necesidad de eliminación de las distintas barreras que obstaculizan la inclusión y la importancia de disponer de los apoyos requeridos para su mejor desarrollo (Bell, 2018).

- Análisis de la definición y del rol actual y perspectivo de la Educación Especial y su relación con la inclusión educativa (Florian, 2013).

- Elevación del reconocimiento social y pedagógico de la inclusión y de sus implicaciones a nivel personal, familiar, institucional y profesional.

No obstante, es válido precisar que cuando la inclusión educativa se realiza sin las condiciones requeridas para su éxito y no se cuenta con el compromiso y la correspondiente preparación de los docentes y de toda la comunidad educativa para su desarrollo, no solo no se pueden apreciar los aspectos positivos antes esbozados, sino que muy probablemente aparecerán situaciones negativas, asociadas por ejemplo, al rechazo de los niños a la escuela por temor al fracaso, a su incomodidad por el tipo de relaciones interpersonales con algunos de sus compañeros, a lo que a veces se une la sensación de frustración de la familia. Ello avala la necesidad de prestar una atención priorizada al desarrollo en los estudiantes de formas positivas de conducta sobre la base del dominio de normas de comportamiento entre todos los compañeros que incluyan el respeto de las normas de comunicación, del turno de palabra, el cumplimiento de las reglas de los juegos en los que participan y el control emocional ante los resultados de las mismos, acompañado de la capacidad para la resolución de manera adecuada de conflictos interpersonales (Sheveleva, 2014).

Por consiguiente, en las condiciones actuales es indispensable tener plena conciencia acerca de que, como titulan Duk y Murillo (2018, p. 11) el editorial del volumen 12 número 1 de la Revista Latinoamericana de Educación Inclusiva "el mensaje de la educación inclusiva es simple, pero su puesta en práctica es compleja". Lo antes dicho no significa que no existan dificultades para la elaboración y difusión del mensaje de la inclusión educativa, en cuya formulación se emplea un mar de conceptos (Bell, 2018), sin que todavía se logre consenso acerca de la forma más adecuada de expresar un mensaje que parece encontrarse más tiempo atrapado en los significados de las palabras que en la expresión y realización, a partir de éstos, de su profundo sentido humano y transformador. En ese contexto, el concepto de necesidades educativas especiales (NEE) no aparenta ser la excepción, como se verá reflejado en el siguiente epígrafe.

\section{Necesidades educativas especiales: concepto, componentes e implicaciones}

A partir del informe Warnock (1978) tuvo lugar la expansión de la utilización del concepto necesidades educativas especiales a escala internacional. Al respecto, Lubovsky (2013) señala que este concepto hace referencia a las necesidades que pueden presentar los estudiantes con o sin discapacidad en el proceso de aprendizaje, para cuya satisfacción se requieren determinadas condiciones particulares que son indispensables para la óptima realización de sus posibilidades actuales y de sus potencialidades. 
Al mismo tiempo, este concepto asume el reconocimiento de la manifestación de las referidas necesidades en diferentes grados, afirmando, a su vez, que por su naturaleza no son homogéneas y que por su carácter pueden ser transitorias o permanentes (López \&Valenzuela, 2015). De acuerdo con De la Oliva, Tobón, Pérez \& Romero (2015) las principales ventajas derivadas de la aplicación del concepto NEE están relacionadas con el interés por lograr la eliminación de términos con una connotación negativa y contribuir a revelar de mejor manera la naturaleza interactiva del proceso educativo y del desarrollo en general. Estos mismos autores resaltan las posibilidades del concepto para contribuir a un conocimiento más profundo de la situación educativa de los estudiantes, que ha de ser asumido como el punto de partida para el diseño de las estrategias educativas, didácticas y metodológicas que respondan efectivamente a sus necesidades y potencialidades. En su análisis acerca de este concepto, Lubovsky (2013) considera las características, las particularidades de funcionamiento e integración de los distintos componentes de las NEE que se resumen en la siguiente tabla:

\begin{tabular}{|c|c|c|}
\hline $\begin{array}{l}\text { Componentes cognitivos- } \\
\text { dominio de las } \\
\text { operaciones mentales }\end{array}$ & Componente energético & $\begin{array}{l}\text { Componente emocional y } \\
\text { volitivo- orientación de la } \\
\text { actividad del estudiante }\end{array}$ \\
\hline $\begin{array}{l}\text { Se relacionan con la } \\
\text { recepción y conservación de } \\
\text { la información percibida. } \\
\text { Incluyen el volumen del } \\
\text { vocabulario, } \\
\text { conocimientos y los } \\
\text { representaciones acerca del } \\
\text { mundo circundante. }\end{array}$ & $\begin{array}{l}\text { Hace referencia a la } \\
\text { activación mental y al } \\
\text { desarrollo de la capacidad } \\
\text { de trabajo de los } \\
\text { estudiantes. }\end{array}$ & $\begin{array}{l}\text { Está determinado por la } \\
\text { motivación, la capacidad de } \\
\text { mantener y concentrar la } \\
\text { atención y por los diversos } \\
\text { mecanismos de reacción } \\
\text { emocional. }\end{array}$ \\
\hline
\end{tabular}

Tabla No. 1 Componentes de las Necesidades Educativas Especiales

Fuente: Basado en Lubovsky (2013)

Al hilo de lo señalado resulta evidente la existencia de múltiples aristas en el abordaje y aplicación del concepto NEE, las que se manifiestan de diversas formas en el proceso de enseñanza aprendizaje, aunque por lo general y de acuerdo con Lubovsky (2013) provocan lentificación en el proceso de formación de conceptos, disminución de la velocidad de recepción, procesamiento y utilización de la información y menor efectividad de los procesos de la memoria. Este mismo autor indica que en ocasiones se presentan retrasos y otros trastornos en el desarrollo del lenguaje y determinadas particularidades en el desarrollo de la actividad mental y de la motricidad. Además, con frecuencia se aprecia también disminución de la capacidad de trabajo de los niños, que suelen agotarse con rapidez.

En este contexto, son crecientes los retos a los que el docente se enfrenta a fin de garantizar las posibilidades para la participación y el máximo aprovechamiento posible de todos sus educandos, poniendo en práctica un amplio arsenal de recursos educativos y didácticos, y garantizando constantemente el apoyo emocional a todos los niños, con énfasis en aquellos en los que se manifiestan determinadas NEE (Bogdanova, 2001) quienes también se encuentran ante grandes desafíos que se derivan de nuevas situaciones comunicativas y de interacción social. Ello subraya la importancia de priorizar el bienestar emocional y unas condiciones de confort psicológico como premisa indispensable para un buen aprendizaje.

A la luz de lo hasta aquí expuesto es posible disponer de valiosos elementos que permiten avalar el paso avance que implicó la introducción y los esfuerzos por lograr la adecuada aplicación del concepto NEE en la práctica educativa, pero la cierta ambigüedad de este concepto, unida al hecho de que no ha desarrollado un aparato categorial propio y al surgimiento de nuevas propuestas en su campo de acción, han comenzado a colocar en la agenda pedagógica actual, la necesidad de su análisis y la posible consideración de nuevas definiciones y conceptualizaciones.

\section{NEE, inclusión educativa e investigación inclusiva}

Siguiendo con la idea final del párrafo anterior, en la actualidad es posible distinguir, además del concepto de NEE, muchos otros que, con sus matices y énfasis propios, aspiran a ser utilizados con mayor éxito y una vida más prolongada en la identificación y atención educativa de los estudiantes que requieren determinadas condiciones adicionales y/o específicas para su exitoso aprendizaje. Entre los referidos conceptos se encuentran personas con diversidad funcional, barreras para el aprendizaje y la participación (Echeita \& Ainscow, 2011), y el que hace alusión a alumno con necesidades y/o alumno con dificultades para la formación (De la Oliva, et al., 2015). Una situación similar ocurre con los constructos educación inclusiva, inclusión educativa, atención a la diversidad o educación para la convivencia, entre otros de los que son utilizados para conceptualizar ese complejo y transformador proceso, en contra 
de cuyas nobles aspiraciones actúan, cada vez con mayor intensidad, factores como la desigualdad y falta de equidad social, que hacen más difícil y al mismo tiempo indispensable la labor de la educación para contribuir a la superación de la exclusión que el impacto de los mismos genera. Como parte de los debates que se producen en torno a estos conceptos, uno de los focos de mayor atención y discusión es el referido a los lugares, es decir, al tipo de escuela a la que debe asistir el alumno con necesidades educativas especiales, sobre todo cuando las mismas están asociadas a una discapacidad.

Nadie niega la importancia de los lugares (Echeita, Muñoz, Sandoval \& Simón, 2014) pero es conveniente tener conciencia acerca de que lo más importante es que el niño acceda y sea protagonista de un proceso educativo de calidad, que responda de manera óptima a sus necesidades y potencialidades. Tal vez en ello radique una de las razones que justifican la continuidad de las incesantes búsquedas de definiciones con sus consecuentes derivaciones prácticas en este campo, que pueden ser ilustradas por el reciente desarrollo de la definición de educación especial inclusiva que, según Hornby (2015) integra las filosofías y prácticas de la educación inclusiva y de la educación especial con la aspiración de educar a los niños con necesidades educativas especiales asociadas a una discapacidad en entornos inclusivos en los que sus necesidades educativas especiales puedan ser satisfechas de un modo efectivo, utilizando los recursos que sean requeridos, junto con las estrategias educativas y metodológicas que conduzcan al más alto nivel posible de inclusión de los estudiantes durante su tránsito por la etapa escolar y al término de la misma. Los apuntes realizados no logran abarcar ni siquiera de manera somera la diversidad de contradicciones e interrogantes que han de ser considerados en los análisis acerca de la inclusión, que se extienden también al ámbito de la investigación, en el que siguiendo a Parrilla (2009) se diferencia la investigación inclusiva de la investigación sobre la inclusión. Así, como subraya Echeita et al. (2014, p. 27) "la distinción nos sitúa, principalmente, ante la relación entre los investigadores y los sujetos de investigación, ante la reflexión sobre la propiedad del proceso y sus resultados y, finalmente, ante los modos y procedimientos para su difusión". La denominada investigación inclusiva aspira a una participación relevante de las personas con discapacidad, que han de intervenir en la misma en calidad de "coinvestigadores" (Echeita et al. 2014), pero, como apunta Bell (2017, p. 204) quedan todavía muchas reservas por explotar en el aprovechamiento y la promoción de: La participación de los estudiantes con discapacidad en la investigación de situaciones y realidades de las cuales ellos son protagonistas y cuentan con vivencias y experiencias que mucho podrían aportar al estudio de la educación de las personas con discapacidad y de la diversidad en general.

Por su parte, la investigación sobre la inclusión está estrechamente relacionada con el nivel metodológico y de desarrollo de su objeto de estudio, lo que condiciona, como precisa Suntsova (2013) la selección de las ideas rectoras para la investigación en esta esfera, la descripción científica de los procesos, hechos y fenómenos educativos para el correspondiente análisis y valoración científica de las características y de la efectividad de las estrategias y procedimientos aplicados. Se trata de investigaciones de naturaleza eminentemente empírica que intentan generalizar diversas experiencias de inclusión.

\section{CONCLUSIÓN}

La exposición de algunos aspectos conceptuales y prácticos de la inclusión educativa desde diversas perspectivas responde al empeño de contribuir al diálogo en favor del análisis y de la identificación de los fundamentos y estrategias que permitan propiciar el necesario avance hacia el logro de los objetivos de este permanente proceso. Para el cumplimiento de ese propósito se ha considerado pertinente la revisión de diferentes fuentes, incluidas aquellas que alcanzan un notable reconocimiento en otras latitudes y a las que comúnmente las barreras lingüísticas obstaculizan su acceso en otros contextos, como es el caso de los países de habla hispana.

Derivado del estudio realizado se han constatado los aspectos positivos de la inclusión para todos los escolares y se han subrayado los riesgos a los que este proceso se enfrenta cuando se acomete su realización sin las condiciones requeridas para su éxito y no se cuenta con el compromiso y la correspondiente preparación de los docentes y de toda la comunidad educativa para su desarrollo. Los elementos aportados acerca de las NEE, la inclusión educativa y la investigación inclusiva reflejan algunos de los principales contenidos del debate conceptual y práctico que tiene lugar en varias de las múltiples problemáticas de la educación inclusiva, que en algún momento dejará de experimentar la necesidad de recurrir a determinados adjetivos para hablar directamente de educación y de investigación, que desde sus diversos paradigmas, enfoques y metodologías están llamadas a contribuir decisivamente a la máxima realización de todos los seres humanos. 


\section{BIBLIOGRAFÍA}

1. Bell, R. (2017). El Desarrollo de los Procesos Sustantivos de la Educación Superior Ecuatoriana ante el Reto de la Inclusión Educativa. Revista Latinoamericana de Educación Inclusiva, 11(1), 199-212.

2. Bell, R. (2018). Inclusión educativa, travesías interminables (2018). Riga: Editorial Académica Española.

3. Богданова, Т. Г. (2001). Специальная дошкольная педагогика и психология: сб. программ нормативных курсов для вузов по спец. 032000-коррекционная педагогика и спец. психология (дошк.). Москва: ВЛАДОС. (Bogdanova, T. G. (2001). Pedagogía y psicología especial preescolar. Compendio de programas de los cursos normativos para las instituciones de educación superior en la especialidad 032000-pedagogía correctiva y psicología especial. Moscú: Vlados.)

4. De la Oliva, D., Tobón, S., Pérez, A. K., \& Romero, J. (2015). El proceso de inclusión social desde la socioformación: Análisis de concepciones sobre discapacidad y necesidades Educativas Especiales. Paradigma, 36(2), 49-73.

5. Duk, C., \& Murillo, F. J. (2018). El Mensaje de la Educación Inclusiva es Simple, pero su Puesta en Práctica es Compleja. Revista latinoamericana de educación inclusiva, 12(1), 11-13.

6. Echeita, G., \& Ainscow, M. (2011). La educación inclusiva como derecho: marco de referencia y pautas de acción para el desarrollo de una revolución pendiente.

7. Tejuelo: Revista de Didáctica de la Lengua y la Literatura, 12, 26-46. Recuperado de https://repositorio.uam.es/handle/10486/661330

8. Echeita, G., Muñoz, Y., Sandoval, M., \& Simón, C. (2014). Reflexionando en voz alta sobre el sentido y algunos saberes proporcionados por la investigación en el ámbito de la educación inclusiva. Revista latinoamericana de educación inclusiva, 8(2), 25-48.

9. Florian, L. (2013). La educación especial en la era de la inclusión: ¿El fin de la educación especial o un nuevo comienzo? Revista Latinoamericana de Inclusión Educativa, 7(2), 27-36.

10. Recuperado de: http://www.rinace.net/rlei/numeros/vol7-num2/art1.pdf

11. Hornby, G. (2015). Inclusive special education: development of a new theory for the education of children with special educational needs and disabilities. British Journal of Special Education, 42(3), 234-256.

12. López, S. \& Valenzuela, G. (2015). Niños y adolescentes con necesidades educativas especiales. Revista Médica Clínica Las Condes, 26(1), 42-51.

13. Лубовский, В.И. (2013). Особые образовательные потребности [Электронный ресурс] // Психологическая наука и образование, 5, 61-66. http://psyedu.ru/journal/2013/5/Lubovskiy.phtml (Lubovsky, V.I. (2013). Necesidades educativas especiales. Recurso electrónico. Ciencia Psicológica y Educación, 5, 61-66.) http://psyedu.ru/files/articles/3989/pdf_version.pdf

14. Назарова, Н.М., Богданова Т.Г, Приходько О.Г., Яковлева И.М. (2018). Педагогика инклюзивного образования. Москва: ИНФРА-М. (Nazarova, N.M., Bogdanova, T.G., Prijodko, O.G., Yakovleva, I.M (2018). Pedagogía de la educación inclusiva. Moscú: INFRA-M).

15. Parrilla, A. (2009). ¿Y si la investigación sobre inclusión no fuera inclusiva? Reflexiones desde una investigación biográfica-narrativa, Revista de Educación, 349, 101-118.

16. Шевелева, Д. Е. (2014). Особенности организации инклюзивного образования в России и за рубежом (компаративистское исследование). Проблемы современного образования, (5), 105-115. (Sheveleva, D. E. (2014). Particularidades de la organización de la educación inclusiva en Rusia y en el exterior (investigación comparativa). Problemas de la educación contemporánea, 5, 105-115.

17. Сигал, Н. Г. (2016). Современное состояние и тенденции развития инклюзивного образования за рубежом. Казань: Казанский (Приволжский) фе-деральный университет. (Sigal, N. G. (2016). Estado actual y tendencias del desarrollo de la inclusión educativa en el exterior. Kazan: Universidad Federal).

18. Сунцова, А. С. (2013). Теории и технологии инклюзивного образования: учеб. пособие. Ижевск: Изд-во Удмуртский университет. (Suntsova, A.S. (2013). Teorías y tecnologías de educación inclusiva: libro de texto. Izhevsk: Editorial de la Universidad de Udmurt).

19. Webster, R., \& Blatchford, P. (2015). Worlds apart? The nature and quality of the educational experiences of pupils with a statement for special educational needs in mainstream primary schools. British Educational Research Journal, 41(2), 324-342. 